\title{
ParFit: a Python-based object-oriented program for fitting molecular mechanics parameters to ab-initio data
}

\author{
Federico Zahariev *1, Nuwan De Silva ${ }^{2}$, Mark S. Gordon ${ }^{3}$, \\ Theresa L. Windus ${ }^{4}$, and Marilú Pérez García ${ }^{5}$, \\ Department of Chemistry and Ames Laboratory, \\ Iowa State University, Ames, Iowa 50011, USA
}
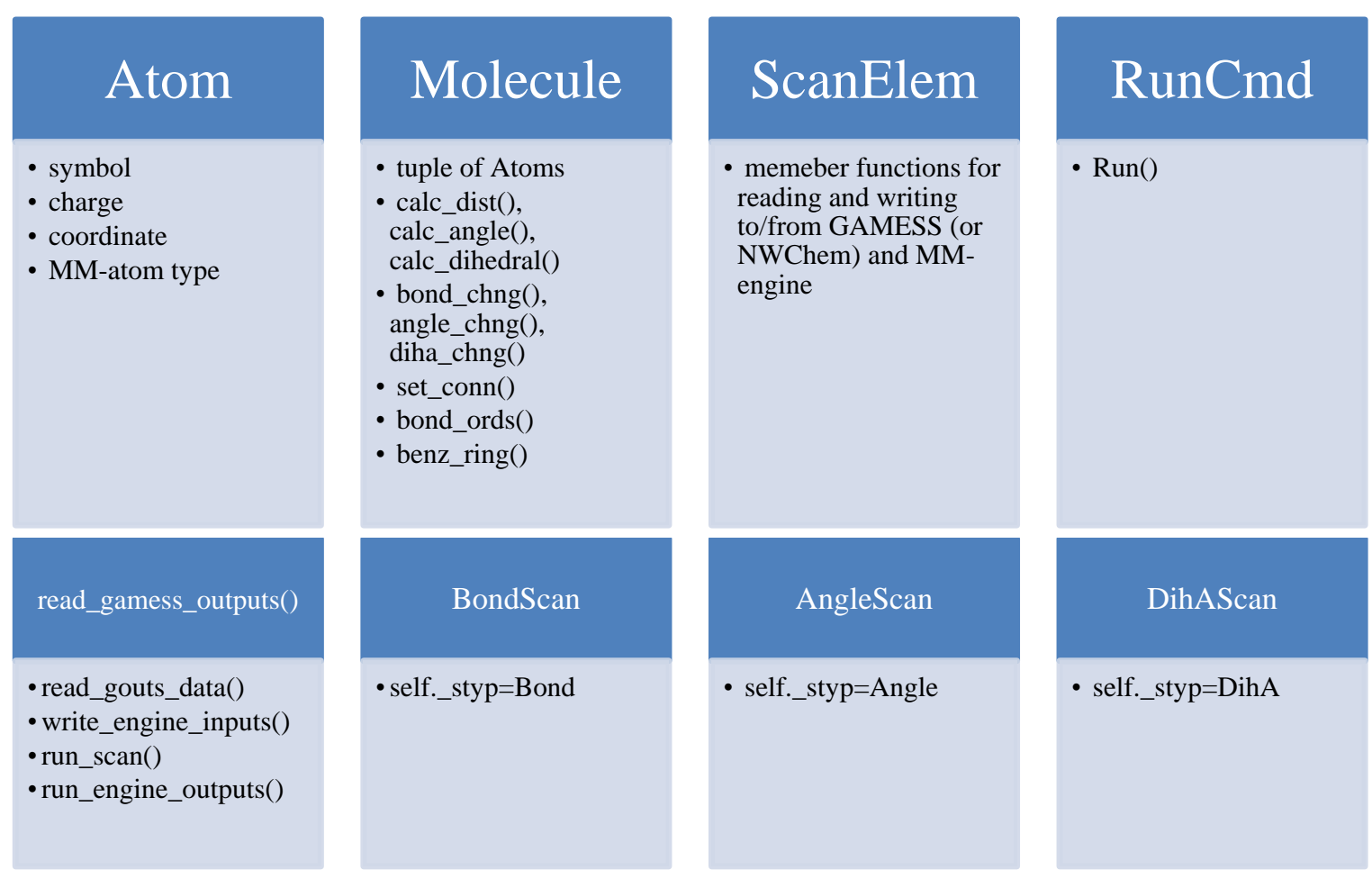

Figure S1. Important class attributes and methods

\section{Illustrative examples of parameter fitting with ParFit}

\footnotetext{
1 fzahari@iastate.edu

2 ndesilva@iastate.edu

3 mark@si.msg.chem.iastate.edu

4 twindus@iastate.edu

5 marilu@iastate.edu
} 
As an example, the ParFit optimization of MM3 parameters for the simultaneous O-P-C*-C*/O-P-C*-H/C-P-C*-C*/C-P-C*-H ("C" means "single-bonded carbon"; "C*" means "double-bonded carbon") dihedral angle rotations of vinyl(dimethyl)phosphine oxide is considered (Fig. S2). Vinyl(dimethyl)phosphine oxide is a representative molecule from a series of organophosphine oxide molecules that were recently studied in ref. 1. The MM3 parameters of vinyl(dimethyl)phosphine oxide were obtained in ref. 1 by manually fitting to MP2 results. In this work, a slightly better quality MM3 parameter fit obtained using ParFit is presented. A hybrid optimization, i.e., an optimization using the genetic algorithm followed by the simplex algorithm, is used in the automatic fitting process.

As a second example, all the MM3 force-field parameters corresponding to the dihedral angles of O-P-C-C, O-P-C-H, C-P-C-C, C-P-C-H in trimethyl-, ethyl(dimethyl)-, and isopropyl(dimethyl)- phosphine oxides, three representative molecules from ref. 1 that are shown on Fig. S4, are simultaneously optimized.
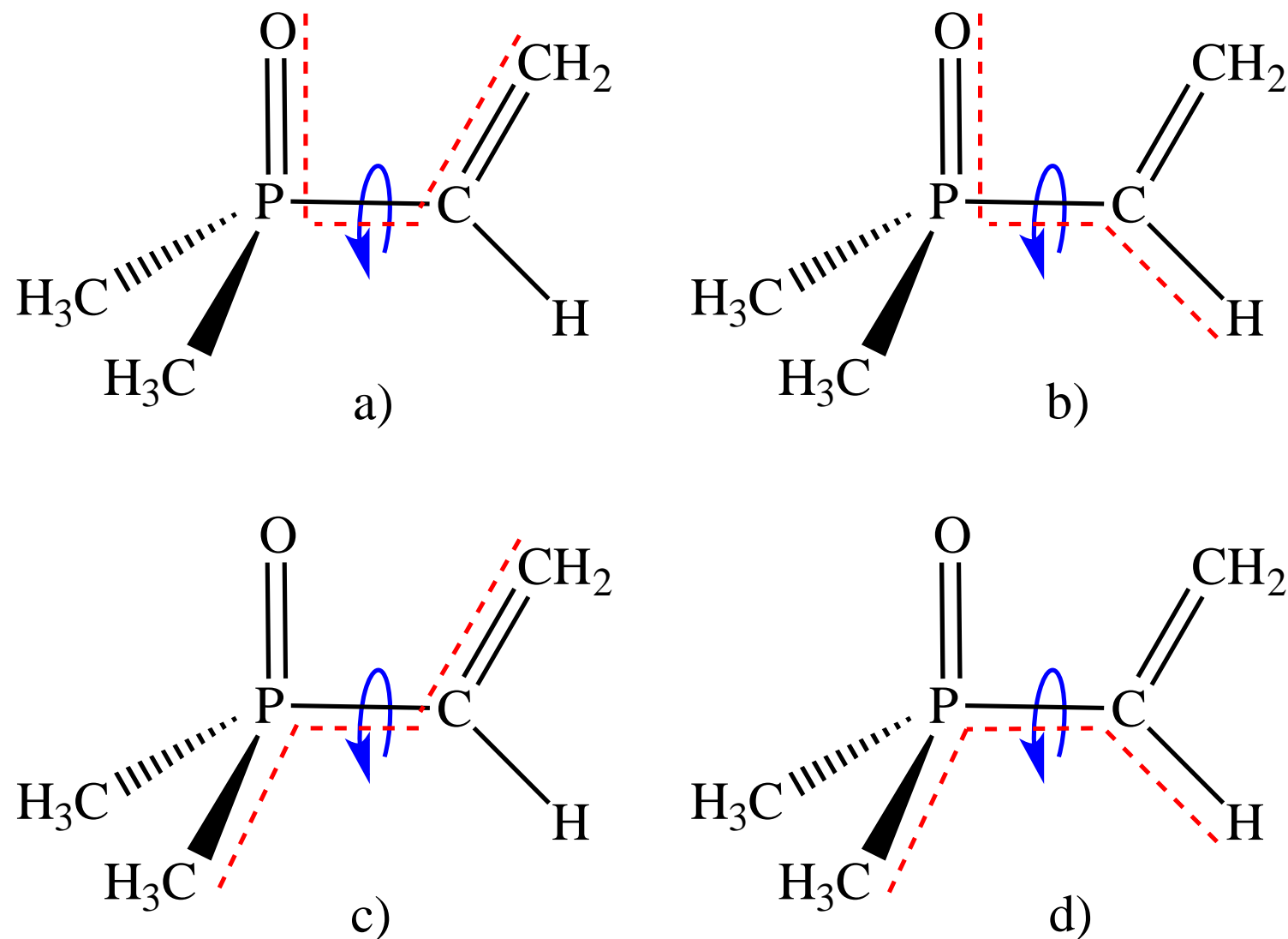

Figure S2. Individual dihedral angles in vinyl(dimethyl)phosphine oxide are highlighted with a dashed red line and defined by: a) O-P-C*-C*, b) O-P-C*-H, c) C-P$\mathrm{C}^{*}-\mathrm{C}^{*}$, and d) $\mathrm{C}-\mathrm{P}-\mathrm{C}^{*}-\mathrm{H}$. The four dihedral-angle rotations are fit simultaneously using ParFit. 
Comparing the energy profiles derived from the initial non-optimized default parameters and the ParFit optimized parameters results in a $2.33 \mathrm{kcal} / \mathrm{mol}$ RMSE improvement when using ParFit. The energy profiles of the default MM3 parameters, with an RMSE of $2.39 \mathrm{kcal} / \mathrm{mol}$, and of the ParFit-optimized MM3 parameters, with an RMSE of $0.06 \mathrm{kcal} / \mathrm{mol}$, are compared in Fig. S3. The ParFit optimization reduces the RMSE by more than an order of magnitude and brings it well below $1.0 \mathrm{kcal} / \mathrm{mol}$.
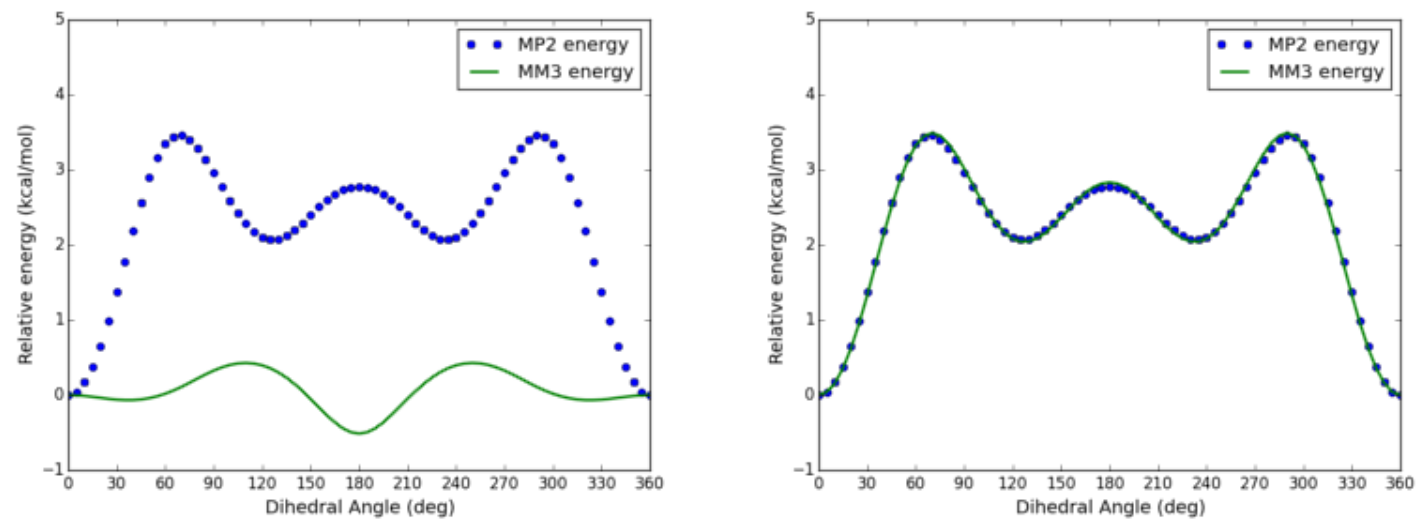

Figure S3. The energy profile of the default MM3 parameters (left) and ParFit-optimized MM3 parameters (right), shown as a green curve, versus MP2 calculations, shown as blue circles, for vinyl(dimethyl)phosphine oxide.

To demonstrated the ParFit capability of handling several molecules at the same time, the simultaneous optimization of MM3 parameters for the O-P-C-C, O-P-C-H, C-P$\mathrm{C}-\mathrm{C}$, and C-P-C-H dihedral angle rotations of trimethylphosphine (Fig. S4a), ethyl(dimethyl)phosphine (Fig. S4b), and isopropyl(dimethyl)phosphine (Fig. S4c) oxides is considered next. 


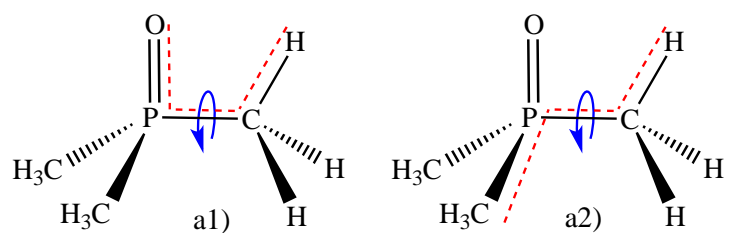

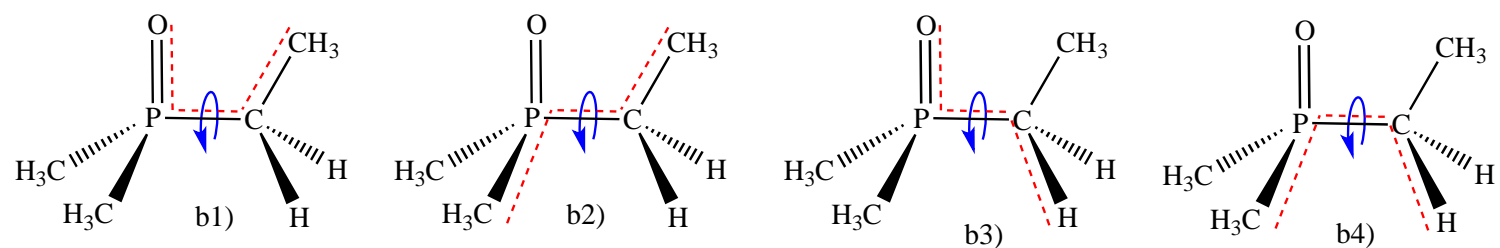

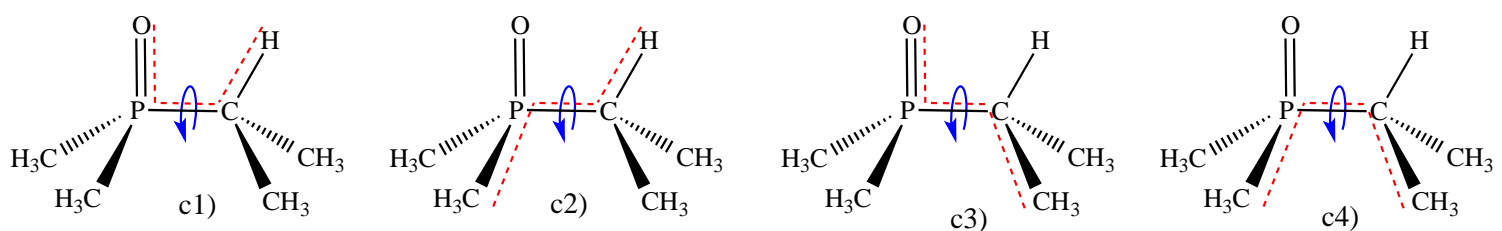

Figure S4. (a) Trimethylphosphine oxide dihedral angles: a1) O-P-C-H, and a2) C-P-C-H. (b) Ethyl(dimethyl)phosphine oxide dihedral angles: b1) O-P-C-C, b2) C-P-C$\mathrm{C}$, b3) O-P-C-H, and b4) C-P-C-H. (c) Isopropyl(dimethyl)phosphine oxide dihedral angles: c1) O-P-C-H, c2) C-P-C-H, c3) O-P-C-C, and c4) C-P-C-C. The dihedral angle parameters for each molecule (a), (b), and (c) are calculated simultaneously using ParFit.

The default and ParFit-optimized MM3 energy profiles of trimethyl-, ethyl(dimethyl)-, and isopropyl(dimethyl)- phosphine oxides are depicted in Fig. S5, Fig. S6, and Fig. S7, respectively. The default/ParFit-optimized RMSE's pairs are $0.04 / 0.03 \mathrm{kcal} / \mathrm{mol}$, $1.18 / 0.08 \mathrm{kcal} / \mathrm{mol}$, and $0.64 / 0.10 \mathrm{kcal} / \mathrm{mol}$, respectively.
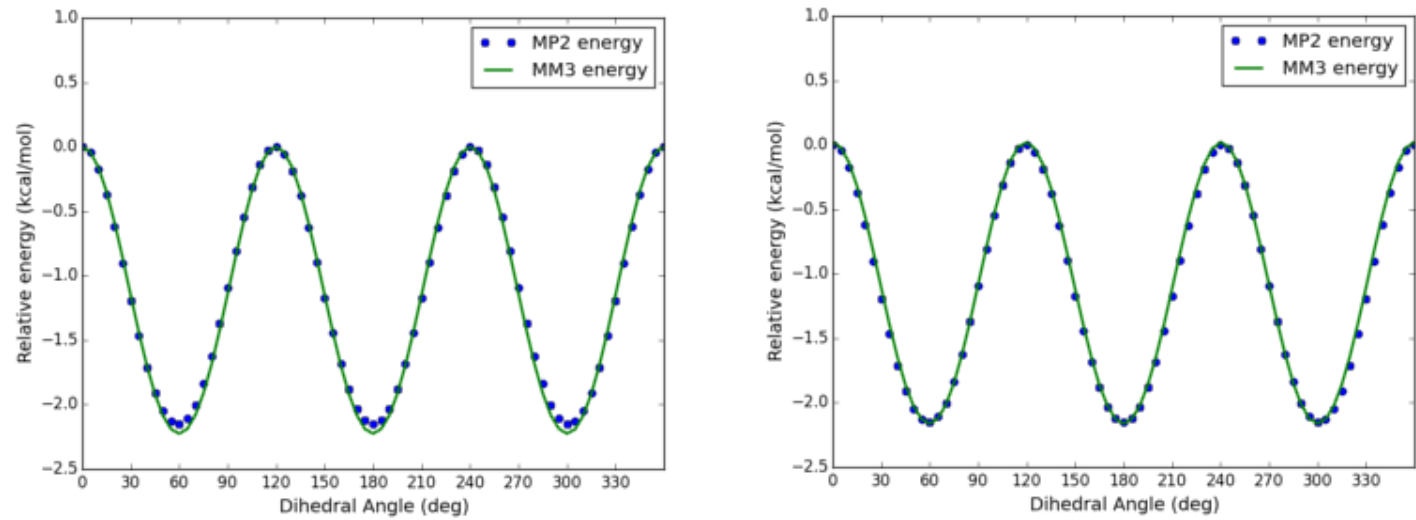

Figure S5. The trimethylphosphine oxide energy profiles of the default (left) and ParFit-optimized (right) MM3 parameters versus MP2 calculations. 

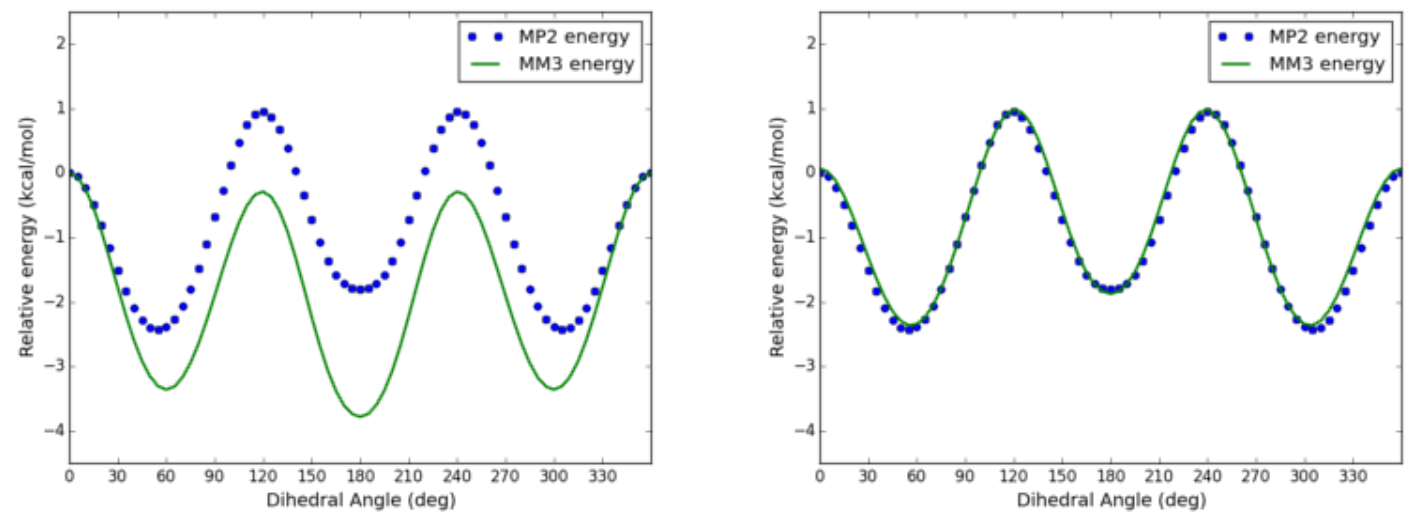

Figure S6. The ethyl(dimethyl)phosphine oxide energy profile of the default (left) and ParFit-optimized (right) MM3 parameters versus MP2 calculations.
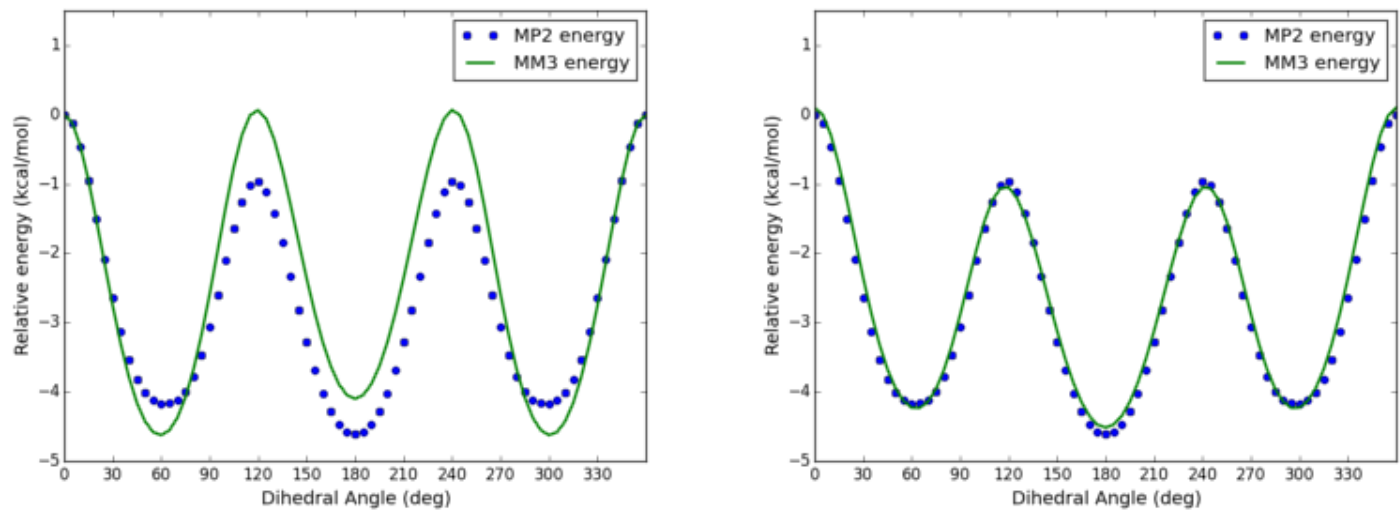

Figure S7. The isopropyl(dimethyl)phosphine oxide energy profile of the default (left) and ParFit-optimized (right) MM3 parameters versus MP2 calculations.

An added benefit of simultaneously optimizing multiple molecules is the improved transferability of the optimized force-field parameters. To demonstrate this feature, the above optimized MM3 parameters are applied to the simultaneous O-P-CC/C-P-C-C dihedral-angle rotational scan data of the tertbutyl(dimethyl)phosphine oxide molecule, yet another representative molecule from ref. 1, (Fig. S8), without any further optimization. 


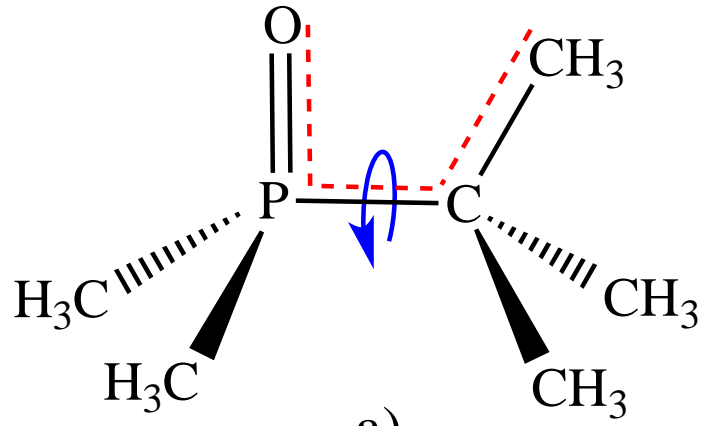

a)

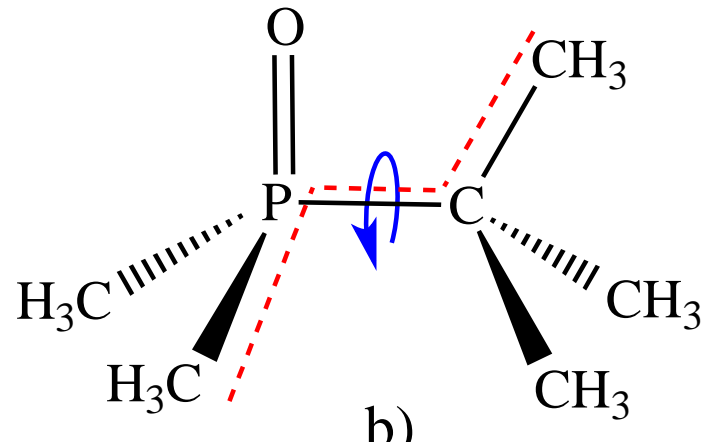

b)

Figure S8. The tertbutyl(dimethyl)phosphine oxide dihedral-angles: a) O-P-C-C, and b) C-P-C-C. The dihedral angles that were used to check transferability of the parameters fit using trimethylphosphine, ethyl(dimethyl)phosphine, and isopropyl(dimethyl)phosphine oxide.

The simultaneously optimized ParFit parameters for tertbutyl(dimethyl)phosphine oxide O-P-C-C/C-P-C-C dihedral angle rotation brings the energy profile RMSE down to $0.12 \mathrm{kcal} / \mathrm{mol}$ from the default $0.60 \mathrm{kcal} / \mathrm{mol}$. The energy profiles comparing default parameters versus the transferable ParFit parameters are shown in Fig. S9.
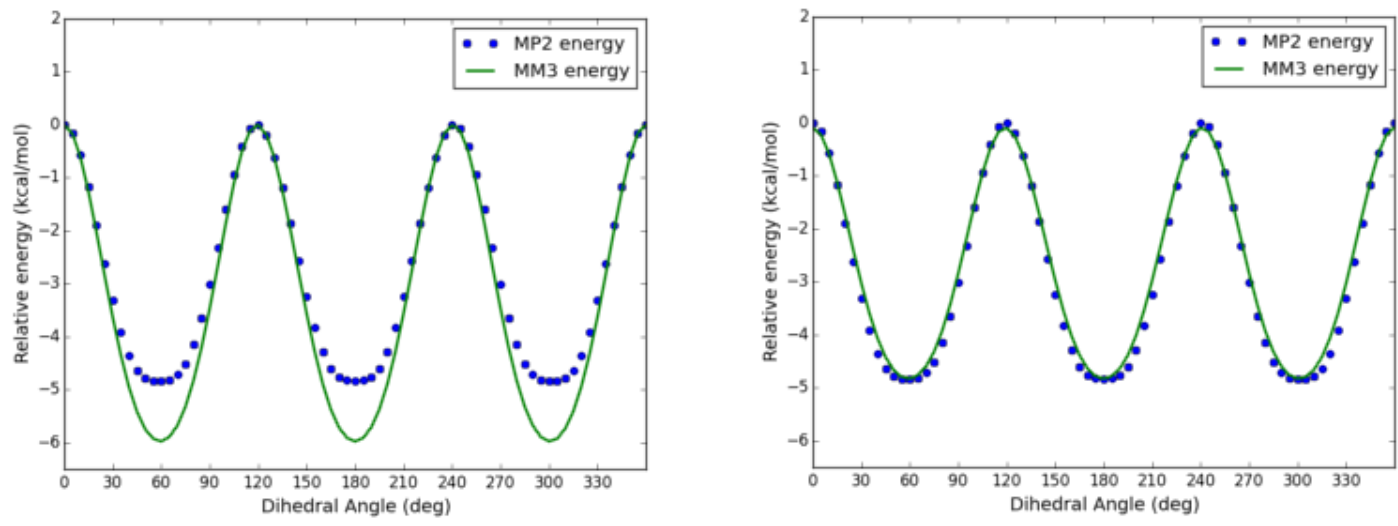

Figure S9. The tertbutyl(dimethyl)phosphine oxide MM3 energy profiles (green) derived from the default parameters (left) and the transferred ParFit parameters (right) as compared to MP2 energies in blue.

An input file for ParFit.py determines the type of scan, i.e. bond, angle, or diha (dihedral); the type of GAMESS (or NWChem) output information, i.e. full (a sequence of GAMESS or NWChem outputs) or comp (a single file for read_gouts_data()); and the base file name. When the QM data is provided in a sequence of output files, the atomic indices for the bond, angle, or dihedral angle, and the range of values in the scan are 
included in the input file. If the QM data is all enclosed within one file, rather than separate output files, the atomic indices and the range of values are read from the comptype file. The input file also contains the path to the directory where the MM engine is installed; the MM type, i.e. $m m 3$ or $m m f f 94$; the fitting algorithm, i.e. fmin, $g a$, or hybr; and the parameters to be optimized. When fitting-parameters are obtained using multiple molecules, as is the case in the example shown in this paper, where dihedral angles in multiple molecules are fitted, the keyword mult is used to specify how many molecules will be used. More details on the ParFit.py input can be found in the documentation that accompanies the code. A utility script $\sim /$ ParFit/Utility/PFinp.py simplifies the creation of a ParFit.py input by allowing the user to answer interactive questions instead of creating the input by hand.

The ParFit input file for the first example is

mult,3

comp, P0101-mp2-popt-dd-

comp, P0102-mp2-popt-dd-

comp, P0103-mp2-popt-dd-

/Users/federicozahariev1/Work/Programs/CODES/MM_engine_source/

$\mathrm{mm} 3$

hybr

208 p p p

$211 \mathrm{ppp}$

$220 \mathrm{p} \mathrm{p} \mathrm{p}$

$223 \mathrm{p} \mathrm{p} \mathrm{p}$

cSV_on

The ParFit input file for the second example is

comp, P0105-mp2-popt-dd-

/Users/federicozahariev1/Work/Programs/CODES/MM_engine_source/

$\mathrm{mm} 3$

fmin

$243 p p p$

$246 p p p$

$249 \mathrm{p} \mathrm{p} \mathrm{p}$

$252 \mathrm{p} \mathrm{p} \mathrm{p}$

cSV_on

(The paths to the MM-engine installation on lines 5 and 2 in the first and second examples, respectively, are machine specific.)

\section{References}

1. De Silva, N.; Zahariev, F.; Hay, B. P.; Gordon, M. S.; Windus, T. L. Conformations of Organophosphine Oxides, J. Phys. Chem. A 2015, 119, 8765-8773. 